Journal of Computer Science 6 (10): 1185-1188, 2010

ISSN 1549-3636

(C) 2010 Science Publications

\title{
Development of a Wall Climbing Robot
}

\author{
Surachai Panich \\ Srinakharinwirot University, 114, Sukhumvit 23, Bangkok 10110, Thailand
}

\begin{abstract}
Problem statement: The problem of a wall climbing robot is holding on the wall. This is challenge for researchers. There are many factors, which effect in holding, all forces, robot movement and mechanical design. Approach: This study proposed movement step design for wall-climbing robot. In design the robot use pneumatic system as main unit to move on the wall. The robot can move in four directions, forward, backward, left and right. Results: We analyzed force acting with the wall that the wall should have only slope from $0^{\circ}$ (parallel with the ground) to $90^{\circ}$ (vertical line). At equilibrium condition, we expressed all forces in equilibrium by sum all forces that equal zero. Conclusion: We can choose parameter to improve the climbing efficiency that the robot can climb much slope. For the first way, we can change the wall material to increase $\mu_{\mathrm{s}}$ and for the second way, we can improve vacuum force by means of increasing pneumatic system efficiency.
\end{abstract}

Key words: Wall climbing robot, force analysis, pneumatic system

\section{INTRODUCTION}

Nowadays, Pneumatic systems use pressurized gases to transmit and control power. Pneumatic systems typically use air rather than some other gas the fluid medium because air is a safe, low-cost and readily available fluid particularly safe in environments where an electrical spark could ignite leaks system components (Gelhaus and Roman, 1990). There are several reasons for considering the use of pneumatic system instead of other systems. Pneumatic systems use air that is exhausted directly into the surrounding environment so this reason is important for selection this system driving the robot (Collie et al., 1990). Control algorithms are an important part, which should be selected by proper with their purposes (Fig. 1).

Computer or microprocessor is used to easily construct control algorithms, but the challenge task is how to communicate between PC or microcontroller and the robot. This is a motive to learn and develop innovation algorithms in order to control robot.

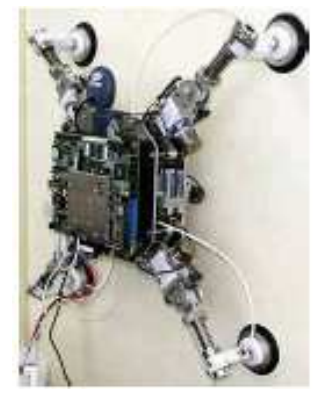

Fig. 1: The example of a wall climbing robot

\section{MATERIALS AND METHODS}

Industrial applications where a vacuum pressure is used include materials handling, clamping, sealing and vacuum forming. In terms of materials-handling applications, a pneumatic vacuum (Collie et al., 1986) can be used to lift smoothly objects that have a flat surface and are not more than several hundred pounds in weight. Figure 2 shows a materials-handling application where a vacuum cup called a suction cup is used to establish the force capability to lift a flat sheet.

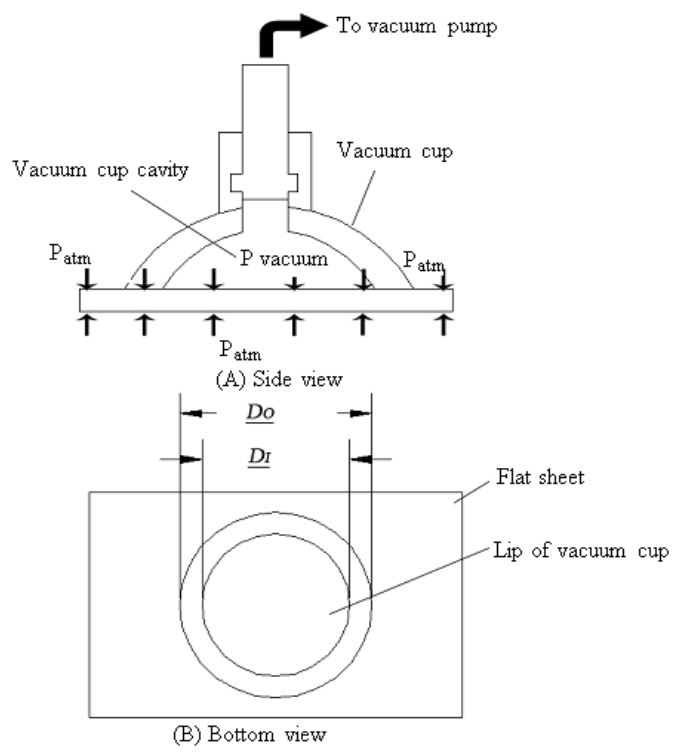

Fig. 2: Vacuum cup used to lift a flat sheet 
The cup is typically made of a flexible material such as rubber so that a seal can be made where its lip contacts the surface of the flat sheet.

Pneumatic vacuum systems: A vacuum pump is turned on to remove air from the cavity between the inside of the cup and top surface of the flat sheet (Watanabe and Kubo, 1992; Yamamoto, 1992). As the pressure in the cavity falls below atmospheric pressure, the atmospheric pressure acting on the bottom of the flat sheet pushes the flat sheet up against the lip of the cup. This action results in vacuum pressure in the cavity between the cup and the flat sheet that causes an upward force to be exerted on the flat sheet. The magnitude of this force can be determined by algebraically summing the pressure forces on the top and bottom surfaces of the flat sheet as follows, as in:

$\mathrm{F}=\mathrm{P}_{\text {atm* }} \mathrm{A}_{0}-\mathrm{P}_{\text {suction* }} \mathrm{A}_{\mathrm{i}}$

Where:

$\mathrm{F} \quad=$ The upward force the suction cup exerts on the flat sheet

$\mathrm{P}_{\mathrm{atm}}=$ The atmospheric pressure in absolute units,

$\mathrm{A}_{0}=$ The area of the outer circle of the suction cup lip

$\mathrm{P}_{\text {suction }}=$ Suction the suction pressure inside the cup cavity in absolute units

$\mathrm{A}_{\mathrm{i}}=$ The area of the inner circle of the suction cup lip

The atmospheric pressure on the top and bottom surfaces of the flat sheet cancels out away from the outer circle area of the cup lip. If all the air were removed from the cup cavity, we would have a perfect vacuum and thus the suction pressure would be equal to zero in absolute pressure units. The exact amount of suction pressure developed cannot be guaranteed and the resulting suction force must be at least as large as the weight of the object to be lifted. Thus a factor of safety is applied with a value of between 2 and 4, depending on the application.

When large, flat sheets are to be lifted, four to eight suction cups are used. In this way the sheet can be lifted uniformly. In addition, the load-lifting capacity is multiplied by the number of suction cups used. When a suction cup is placed on the top of a flat sheet and the vacuum pump is turned on, a certain amount of time must pass before the desired vacuum.

Wall-climbing robot design: The problem, how to holding on the wall is solved by many methods. There are many factors, which effect in holding, all forces, robot movement and mechanical design.

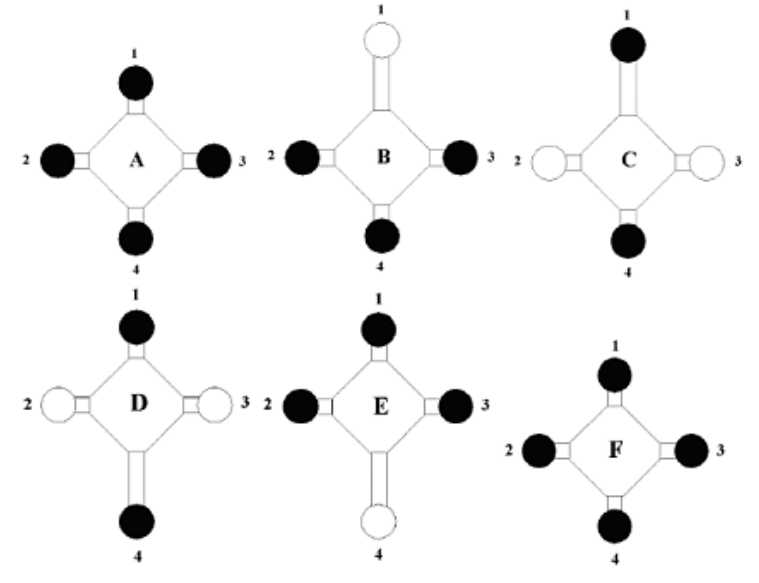

$\bigcirc$ Vacuum cup as air-injection line

Vacuum cup as air-suction line

Fig. 3: Movements steps of the robot in forward direction

Movement step design: Robot movement is carefully designed because the robot moves on the wall. The robot must move and hold on the wall in the same time. From this constraint, a robot movement is designed as shown in Fig. 3. It shows six movement steps only in forward direction, but other directions; left, backward and right directions are similar as forward movement steps. As the robot is moving, in each step, robot must remain minimum two vacuum cups to hold on the wall. In designing, robot can move in line, which is controlled by pneumatic cylinders. Figure 3 shows a pattern of robot movement to forward direction for a cycle step by starting from alphabet ordering (A) to (F). Frame (A) shows the initial robot condition and will start movement in forward direction.

In frame (B) robot stops air-suction line and changes to air-injection line in vacuum cup 1 and robot lifts the vertical cylinder with vacuum cup 1 then contract parallel cylinder 1 then releases the vertical cylinder 1 with vacuum cup 1 and then expand vertical cylinder 1 and final step vertical cylinder 1 moves in original position and changes to air-suction line again. In frame (C) robot stops air-suction line and changes to air injection line in vacuum cup 2 at left side and in vacuum cup 3 at right side. Then the robot lifts both the vertical cylinder 2 and vertical cylinder 3 . In frame (D), robot contracts parallel cylinder 1 and expands parallel cylinder 4 in the same time (robot moves its body to new position from back to forward). In frame (E), robot stops air-injection line and changes to air- suction line 
in vacuum cup 2 at left side and in vacuum cup 3 at right side. And then it stops air-suction line and changes to air-injection line in vacuum cup 4. In frame $(F)$, robot contracts parallel cylinder 1 and expands parallel cylinder 4 in the same time (robot moves its body to new position from back to forward). The robot moves to the original position and prepares for next instruction from PC. In the other directions (left, right, backward), movement steps are similar as above pattern.

\section{RESULTS}

The force analysis is guarantee that the robot can hold and move on the wall. We will analyze force acting with the wall that the wall should have only slope from $0^{\circ}$ (parallel with the ground) to $90^{\circ}$ (vertical line). All forces are acting on the slope wall can show in free-body diagram. Figure 4 shows free-body diagram that consists of all forces, vacuum force, reaction force, robot weight and friction force. The vacuum force is exerted by pressure difference between atmosphere pressure and inside vacuum cup pressure. The robot weight is force, which depends on the robot Mass (M) and acceleration of gravity (9.81 $\mathrm{m} \mathrm{sec}^{-2}$ ), which has downward direction. Friction force is due to the irregularities of the surfaces in contact. At equilibrium condition, we express all forces in equilibrium by sum all forces that equal zero. We thus obtain the following two equilibrium equations from equation 2 and 3, which express, respectively, that the sum of the X-Component and the sum of the $\mathrm{Y}$ Component of given forces must be zero:

\section{$\sum F y=0$}

Recation force - vacuum force - robot weigth $=0$

$\sum \mathrm{Fx}=0 ;$ Robotweight - friction force $=0$

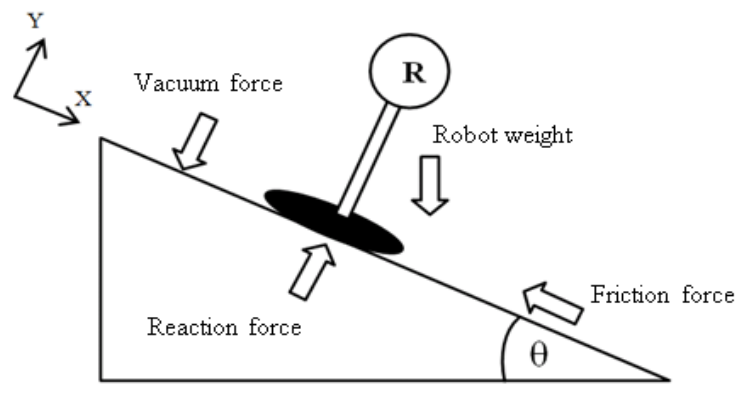

Fig. 4: All forces acting on the sloped wall
Regarding free-body diagram, all forces exerted by robot will be resolved into $\mathrm{X}$ and $\mathrm{Y}$ component. We first determined vacuum force. From Fig. 4 and Eq. 2-3, we can calculate vacuum force to hold robot on the vertical terrain by below steps. The suction pressure calculation equals:

Psuction $_{(\mathrm{abs})}=$ Psuction $_{(\mathrm{gage})}+\mathrm{P}_{\mathrm{atm}}$

The vacuum force calculation, which can be found using Eq. 4:

$\mathrm{F}_{\text {vacuum }} * \mathrm{P}_{\text {atm. }} \mathrm{A}_{0}-\mathrm{P}_{\text {suction (abs). }} \mathrm{A}_{\mathrm{i}}$

The friction force is calculated by Eq. 2-5:

$\mu_{\mathrm{s}}\left(\mathrm{F}_{\text {vacuum }}+\mathrm{Mg} \cos \theta\right)=\mathrm{Mg} \sin \theta$

From Eq. 6, shows friction force that should be theoretically equal the robot weight, in order to the robot can hold on the wall. But we should construct the robot that has robot weight less than friction force, because we do not know some unknown forces. If robot weight in $\mathrm{X}$-axis is less than friction force (robot weight cannot overcome maximum friction force), robot will not slide to ground, but still hold on the wall with a vacuum cup. From robot weight in $\mathrm{X}$-axis, it equals $\operatorname{Mg}(\operatorname{Sin} \theta)$ and below analysis. So the equations are:

$\mu_{s}\left(F_{\text {vacuum }} \operatorname{Mg}(\operatorname{Cos} \theta)\right) \geq \operatorname{Mg}(\operatorname{Sin} \theta)$

and:

$\mu_{\mathrm{s}} \geq \frac{\operatorname{Sin} \theta}{\left(\left(\mathrm{F}_{\text {vacumm }} / \mathrm{M}_{\mathrm{g}}\right)+\operatorname{Cos} \theta\right)}$

From Eq. 8, we can choose parameter to improve the climbing efficiency that the robot can climb much slope.

\section{DISCUSSION}

Now we do not consider uncertain force when the robot is on the wall such as vibration force as movement and others. Not consider all moments when the robot is on the wall, because the piston rod of pneumatic cylinder is made from strength material.

\section{CONCLUSION}

This study has proposed movement step design for wall-climbing robot. 
In design the robot use pneumatic system as main unit to move on the wall. The robot can move in four directions, forward, backward, left and right. During the robot move on the wall, the robot must remain always the vacuum cups for holding. We can choose parameter to improve the climbing efficiency for hardware design. The first way, we can change the wall material to increase $\mu_{\mathrm{s}}$. The second way, we can improve vacuum force or reduce robot weight $\left(\mathrm{M}_{\mathrm{g}}\right)$, by using robot light material, example straight plastic. This study is valuable as literature review for researchers, who want to construct wall-climbing robot.

\section{ACKNOWLEDGEMENT}

This research from Measurement and Mobile Robot Laboratory ( $\mathrm{M}$ and M-LAB) was supported by Faculty of Engineering, Srinakharinwirot University.

\section{REFERENCES}

Collie, A.A., L. Billingsley and L. Hatley, 1986. The development of pneumatically powered walking robot base. Proceeding of the IMechE C371/86 Conference, Dec. 1986, ImechE, c377/86 Pub, London, pp: 137-144.
Collie, A.A., J. Billingsley and E. von Puttkamer, 1990. Design and performance of the Portsmouth climbing robot. Proceeding of 7 th International Symposium on Automation and Robotics in Construction, June 1990, Bristol, England, pp: 125-130.

Gelhaus, F.E. and H.T. Roman, 1990. Robot applications in nuclear power plants. Nuclear Energy, 23: 1-33.

Watanabe, A. and K. Kubo, 1992. Development of an automatic inspection robot for nuclear power plants. Ind. Robots, 19: 25-29.

Yamamoto, S., 1992. Development of inspection robot for nuclear power plant. IEEE Int. Conf. Robot. Automat., 2: 1559-1566. 